ISSN: 2386-3919 - e-ISSN: 2386-3927

DOI: https://doi.org/10.14201/et20193716181

\title{
ANÁLISIS DE ELEMENTOS PARA LA DEFINICIÓN \\ DE UN MODELO DE SUPERVISIÓN ESCOLAR EN EDUCACIÓN DE PERSONAS ADULTAS
}

\author{
Analysis of elements to define a school supervision paradigm in \\ Adult Education
}

Jesús Manuel LuCEndo PATiÑO

Universidad Nacional de Educación a Distancia-UNED

Facultad Educación. Madrid. España

jlucendo3@alumno.uned.es

bttps://orcid.org/0000-0003-0738-576X

Recibido: 15/04/2019; Aceptado: 10/05/2019; Publicado: 01/06/2019

Ref. Bibl. JESÚS MANUEL LUCENDO PATIÑO. Análisis de elementos para la definición de un modelo de supervisión escolar en Educación de Personas Adultas. Enseñanza \& Teaching, 37, 1-2019, 61-81.

RESUMEN: El objetivo principal de esta investigación consiste en analizar las características y elementos necesarios para la definición de un modelo de Supervisión Escolar en Educación de Personas Adultas que responda a las necesidades y demandas de aprendizaje de las personas y las colectividades adultas actuales, considerando, desde una perspectiva holística del sistema educativo, la Inspección de Educación un factor clave para la mejora y la calidad pedagógica y la Educación Permanente una modalidad de enseñanza estratégica y de creciente relevancia para el desarrollo de la sociedad del siglo XxI.

Metodológicamente, en el marco teórico del Paradigma Comprensivo, se adopta el Método de Comparación Constante de la Teoría Fundamentada de Glaser y Strauss, utilizando como instrumento de recogida de información la técnica de entrevista estructurada en profundidad y seleccionando los informantes mediante muestreo no probabilístico intencionado con coeficiente experto, $\mathrm{k}>0.8$. El estudio de datos se realiza utilizando el programa de análisis cualitativo ATLAS.ti para la codificación y elaboración de metavariables.

A partir de esta categorización se concluye la prevalencia de un modelo de supervisión no burocrático, orientado a la mejora y calidad educativas, que atribuye 
nuevas funciones de liderazgo, coordinación y participación en la planificación a la Inspección de Educación, además de las tradicionalmente desempeñadas de evaluación, control y asesoramiento. Se diseña una red conceptual para configurar un modelo de supervisión educativa en Educación de Personas Adultas basado en la valoración criterial y el asesoramiento a centros y profesorado más que en el control, con técnicas de supervisión colaborativas que focalizan la atención sobre la metodología, la práctica docente y el establecimiento de objetivos para la mejora continua.

Palabras clave: supervisión escolar; educación de personas adultas; inspección educativa; educación permanente; organización escolar.

SUMMARY: The main objective of this research is to study all the needed features to develop a school supervision model in Adult Education that meets the learning requests of adult students, considering, from an educational system holistic perspective, the Education Inspectorate as a key factor to improve the pedagogical quality, and the lifelong learning a strategic point in the XXI century.

The research method used is the Constant Comparative System of the Glaser and Strauss Theory, framed in the Comprehensive Paradigm. The structured in-depth interview is applied to data collect. Interviewees are selected according to not intentional probabilistic sampling with expert coefficient, $\mathrm{k}>0.8$. The data handling is done using the qualitative analysis program ATLAs.ti by means of the codes and metavariables elaboration.

Based on this categorization process the prevalence of a non-administrative supervision model is concluded. It must be oriented towards educational improvement and quality and it should assume new functions such as leadership, coordination and participation in educational planning, in addition to those traditionally assigned such as evaluation, control and advice. Furthermore, the study contains a conceptual network designed to configure a paradigm of educational supervision in Adult Education based on the criterial assessment along with schools and teachers counseling rather than in bureaucratic control, with collaborative monitoring techniques that focus attention on methodology, learning training and setting objectives to achieve a continuous improvement in teaching practice and school management.

Key words: school supervision; education inspectorate; adult education; lifelong learning; school management.

\section{INTRODUCCIÓN}

El propósito primordial de esta investigación consiste en analizar los elementos y características necesarios para diseñar un modelo de supervisión escolar en enseñanzas de Educación de Personas Adultas que responda a los cambios en las demandas y necesidades del sistema educativo y la sociedad del siglo XXI.

Asumiendo la visión recabada en la literatura académica relativa a este ámbito (Gento, 2004; Camacho, 2014; Campos, 2017; Ehren, 2017; Ramírez, 2017; Secadura, 
2014; Vázquez-Cano, 2017; Martín, 2013) que entiende el papel de la Inspección de Educación como un elemento esencial en la mejora escolar y la calidad educativa si se le atribuye un posicionamiento y funciones adecuadas, consideración que tiene una transcendencia especial en el ámbito de la Educación de Personas Adultas dada la mayor flexibilidad y menor regulación de estas enseñanzas, el diseño de investigación plantea realizar un análisis de la situación de la Inspección de Educación, las necesidades a las que debe responder y que han de caracterizar la Educación de Personas Adultas en la actualidad, para determinar los rasgos que deben definir un paradigma de supervisión educativa que pueda orientar la intervención de la Inspección de Educación en esta tipología de enseñanzas con una mayor satisfacción por parte de la comunidad educativa, eficacia organizativa y calidad pedagógica. Se proyecta formalizar la configuración de los servicios de inspección para, una vez categorizadas las funciones y los quehaceres de los inspectores de educación estudiar su incidencia como agentes de mejora en los centros docentes de EPA, Educación de Personas Adultas.

Necesidad de un modelo de supervisión avalada por Casanova (2015), que afirma "conseguir modelos apropiados para el desarrollo y aplicación de las competencias supervisoras, supone, en buena medida, garantizar la calidad del sistema educativo" (p. 16).

La justificación y pertinencia académica de la investigación toma como punto de partida el estudio de la literatura científica en ambos ámbitos. Como primera cuestión, citar que la fundamentación teórica de la Supervisión Educativa deriva de paradigmas pedagógicos u organizativos genéricos y adaptados, puesto que es un ámbito de estudio con poca producción en investigación y publicaciones, especialmente en nuestro país, con un corpus teórico limitado frente a otros campos de conocimiento como la dirección de centros o la orientación escolar. En palabras, que corroboran esta oportunidad de la investigación, de Silva (2013: 69):

Se observan pocos estudios sobre estrategias relacionadas con el papel de la inspección escolar orientados a impulsar acciones organizativas en la zona escolar y propuestas que incidan en la mejora de los resultados académicos. Esta orientación contrasta con las recomendaciones que hacen los expertos respecto a la necesidad de realizar pesquisas acerca de los agentes que intervienen directamente en los centros educativos y fomentar la investigación en este campo.

Salgado (2009) ratifica que "son muy escasas las investigaciones que tienen por objeto describir los contextos en los que se desenvuelve el trabajo de los inspectores. Quiero decir faltan estudios rigurosos relacionados con la realidad en la que los inspectores desenvuelven su realidad" (p. 26). Miguel et al. (2018) exponen la necesidad de realizar estudios académicos y de investigación en todo el ámbito de la Unión Europea cuando afirman que «los estudios europeos de Eurydice, la Red de información sobre educación de la Comisión Europea, abordan la inspección educativa de forma tangencial» (p. 131), añadiendo que "hasta el momento la Red Eurydice no ha considerado la inspección educativa como objeto de estudio, sino 
que se ha referido a ella al tratar la evaluación de distintos aspectos de los sistemas educativos» (p. 133).

En segundo lugar, considerar que las demandas de la sociedad en el siglo XXI al sistema educativo son diferentes en muchas ocasiones a las de la centuria pasada y que los centros escolares, los profesionales de la educación y los alumnos han cambiado sus planteamientos, lo cual se incrementa si consideramos el ámbito de la Educación de Personas Adultas, en el marco del Aprendizaje Permanente, con objetivos y metodologías novedosos derivados de los cambios sociales y tecnológicos, así como el desarrollo de las nuevas tecnologías e Internet.

Existe abundante literatura académica (Fernández Enguita, 2016; Estefanía, 2014; Fullan, 2002) respecto a que estamos en una época de cambio en la cual la sociedad reclama cada vez más exigentes demandas en mejora y calidad al sistema educativo, más apertura y originales respuestas de aprendizaje que se adapten a las nuevas necesidades de los ciudadanos del siglo XXI.

En este contexto, "la educación de las personas adultas se ha convertido en un instrumento formativo central en la actual sociedad global” (Rumbo, 2016: 105), siendo especialmente sustancial dotarla de relevancia con una configuración propia en el ámbito de la Educación Permanente (Sanz, 1998; Alonso, 2002; Consejo de la Unión Europea, 2011), diseñando una didáctica y una estructura organizativa específicas para la Educación de Personas Adultas (López-Barajas y Sarrate, 2002), garantizando la educación como un bien común a lo largo de toda la vida (UNESCO, 2015a).

"La teoría y práctica de la educación de adultos debe conducir hacia nuevas ocasiones y nuevas formas de participación e incorporación cultural de las personas adultas en todo el entramado social y comunitario» (García y Martín, 2007: 35) que transciendan el carácter compensatorio y remedial de los centros de Educación de Adultos, cuya configuración básica los cataloga actualmente como escuelas de segunda oportunidad (Lancho, 2007), en contradicción con los planteamientos teóricos vigentes en Pedagogía y las necesidades impuestas por el cambio de nuestra sociedad sobre la necesidad de Educación Permanente y Formación a lo largo de la Vida (Jiménez, 2009). Su potencialidad sería mucho mayor si se abren a su entorno y toman contacto con instituciones y profesionales que ejerzan el liderazgo social con un enfoque más proactivo, flexible y adaptado.

La Educación de Adultos puede constituirse en una estrategia clave para abordar muchos de los retos de nuestra Europa actual si se orienta a responder adecuadamente a las necesidades exigidas (European Commission, 2017). La UNESCO (2016: 3) es categórica en este sentido afirmando: «En Europa operan una gran variedad de programas de educación y formación sobre competencias básicas para adultos, es necesaria más investigación en esta área [...] Monitorizar y evaluar la Educación de Adultos es vital»; incidiendo la Comisión Europea (European Commission, 2015) en la relevancia de la evaluación como factor garante de calidad en estas enseñanzas.

Este nuevo marco social y pedagógico confluye en la supervisión educativa como un elemento situado en la cúspide del sistema escolar con una enorme capacidad de influencia, de orientar e intervenir en las comunidades educativas 
desde un posicionamiento revestido de la "auctoritas» específica propia del cuerpo, de conocimiento experto de sus miembros y con una visión global, pero cercana al mismo tiempo a la práctica docente. La supervisión educativa se presenta como una tarea necesaria en el ámbito de la Educación de Personas Adultas, tanto más cuando se puede considerar una tipología de enseñanza más requerida de flexibilidad y dinamismo, menos ordenada que la enseñanza ordinaria y con una población escolar más heterogénea. Supervisión Escolar y Enseñanza de Adultos se necesitan ineludiblemente: la Inspección debe ejercer un papel decisivo en la transformación de la escuela a la par que los procesos de innovación y cambio necesitan ineludiblemente de supervisión y evaluación (Estefanía, 2014; Vázquez-Cano, 2017).

En los últimos años, en la propia actuación profesional y en la literatura académica, se demanda con frecuencia dotar de más relevancia, consideración y peso social a la Inspección de Educación (Camacho, 2014; Alcalá, 2016; Gaertner y Pant, 2011; Secadura, 2014) como un elemento fundamental del sistema educativo para asegurar la calidad y la mejora (Campos, 2017). Es «una pieza fundamental en todo sistema y en toda Administración Educativa" (Mayorga, 2000: 193), aunque también en este paisaje en transformación es indispensable repensar globalmente el rol y la posición de los inspectores en la organización académica, al igual que sus relaciones con los centros, reconceptualizar el enfoque y las actuaciones supervisoras (Rul, 2013), orientándolas permanentemente a la mejora de la calidad educativa (Martín, 2013; OfSTED, 2017a; Ramírez, 2017; Gento, 2004). Secadura corrobora esta visión (2017: 489):

La realidad es que, en nuestros días, la Inspección Educativa tiene una identidad débil, sin un claro contenido propio, y el perfil profesional del inspector lo apreciamos permanentemente incierto, sin la suficiente definición. Así, en la actualidad, observamos que la contradicción esencial está en delimitar cuál es, o cuál debe ser, el referente de la Inspección Educativa del siglo XXI.

Renovadoras teorías en Supervisión Escolar como el modelo de Gestión de Calidad Total (Bradley, 1993), que preconiza el paso de una supervisión lineal a cíclica para la mejora continua de manera holística, o el modelo de generación de expectativas previas y retroalimentación mediante redes policéntricas (Ehren, 2017); innovadores paradigmas de liderazgo como el Modelo Deming (Gento, 2001); modelos de supervisión basados en proyectos (Silva, 2013); paradigmas de cambio del foco evaluador del «output» al «outcome» (Mills-Scofield, 2012); propuestas de internacionalización de la inspección (Ramírez, 2017); experiencias de gestión escolar como los "contratos objetivos» en Francia, "contratos de autonomía» en Portugal o el programa de entrenamiento colaborativo en Escocia; nuevas tipologías de supervisión como la diferenciación entre supervisión preventiva, supervisión constructiva o supervisión creadora (Campos, 2017); conceptos como el del "amigo crítico" (MacBeath, Schratz, Meuret y Jacobsen, 2000); la incorporación de Cartas Éticas de Servicios y Deontológicas (Vázquez-Cano, Arévalo y Gómez de Agüero, 2011); planes estratégicos de cooperación internacional entre Servicios de Inspección de 
Educación (IGEN, 2016; SICI, 2016) abren un amplio abanico de perspectivas en supervisión educativa y enseñanza de adultos.

Existe una tendencia a considerar que el inspector debe "trabajar con" en lugar de «intervenir en» (Domingo, 2001), en centros que aprenden para la mejora continua (Bolívar, 2000); transitar desde la situación actual, externa a los centros, documentalista, con técnicas y funciones apegadas a su devenir histórico, hacia un paradigma de servicio público (OFSTED, 2017b), garante del derecho a la educación de calidad para todos, durante toda la vida, priorizando la equidad e inclusión educativas (Gento, 2004; UnEsCo, 2015b; Matthews y Sammons, 2004; Aguerrondo, 2013). Sintetizando con palabras de Casanova (2015: 9):

Hace algunas décadas se hablaba de la supervisión como garante para que la educación de calidad llegara a todos los niños y niñas de un país. Como se puede observar, hemos cambiado el término "niño" por el de "persona». Las características de la sociedad actual [...] han llevado a que la educación permanente se convierta en una de las finalidades de cualquier sistema educativo institucional [...] No se puede olvidar desde el enfoque de la supervisión, que tan importantes son las etapas educativas obligatorias como las post-obligatorias, incluso, como digo, las que se ofertan a personas adultas. En estos momentos, este es un buen campo de trabajo para la supervisión.

\section{Metodología}

El estudio se fundamenta teóricamente en el Paradigma Comprensivo (Cardona, 2013), cuya finalidad es establecer desde un análisis en profundidad la necesidad de dar respuesta a las novedosas variables de cambio y transformación derivadas de los fenómenos (globalización, nuevas tecnologías, etc.) que definen la impronta de nuestro tiempo, desde un posicionamiento de partida ecléctico y de conciliación crítica. En el procedimiento de investigación se utiliza una metodología inductiva exploratoria (Sevillano y Vázquez-Cano, 2015) basada en la obtención de información y datos a partir de los cuales se pueda dar cumplimiento al objetivo de la investigación, generar códigos y categorizaciones que puedan fundamentar la caracterización del modelo.

Los objetivos propuestos pretenden buscar fundamentación teórica y metodológica a la actuación inspectora desde el punto de vista del saber académico y el conocimiento universitario, estableciendo indicadores de constructo; conocer la existencia y características de modelos de supervisión educativa para la educación de adultos ya definidos y validados; recoger información sobre paradigmas pedagógicos de organización educativa que puedan dar sustento científicamente al modelo en desarrollo; indagar experiencias previas de investigación en el ámbito de las temáticas del presente estudio; rastrear la búsqueda de literatura científica, materiales, herramientas e instrumentos novedosos; realizar una aproximación investigadora sobre la relación de la Universidad con la Inspección de Educación y la Enseñanza de Personas Adultas; por último, conocer opiniones y experiencias, 
recabando valoraciones críticas desde el punto de vista de expertos de los ámbitos académicos y profesional sobre los elementos básicos de este modelo.

El modelo teórico seguido está basado en el planteamiento diseñado por Glaser y Strauss (1967) denominado Grounded Theory o Teoría Fundamentada. Según Sandoval (1997), la Teoría Fundamentada «es una metodología general para desarrollar teoría a partir de datos que son sistemáticamente capturados y analizados». La característica principal de este método reside en que la construcción de las hipótesis, conclusiones y modelos se realiza directamente desde los datos y no desde marcos teóricos preexistentes mediante el Método de Comparación Constante (MCC), lo cual implica una continua revisión y comparación de los testimonios obtenidos para ir construyendo teorías desde la realidad en un proceso continuo de circularidad sistémica. Para elaborar las teorías y modelos es fundamental que se elaboren códigos para categorizar la información, así como que se construyan y relacionen las categorías encontradas que constituyen el elemento conceptual de la teoría y muestran las relaciones entre las hipótesis y los datos. Una última característica de este paradigma metodológico es su gran fuerza en modelización y visualización analíticas: genera trazados de esquemas gráficos que, además de ayudar en el análisis de datos y la construcción de la teoría, permite una presentación de la información explícita.

Puesto que el objetivo básico de la presente investigación consiste en la elaboración de un modelo de supervisión escolar en Educación de Personas Adultas, es conveniente definir y delimitar el concepto. Fernández-Pérez (1978) considera que «modelo es una explicación de una realidad basándose en una teoría previa ya preestablecida, es una construcción teórica que intenta simplificar y organizar la realidad» (p. 34). Medina (2012) afirma:

En el campo de la ciencia, el modelo teórico es una construcción intelectual que se realiza con la intención de explicar algo o de abordar algún problema. Aunque el modelo no coincide exactamente con lo que es una teoría, sin embargo, comparte con esta su finalidad descriptiva y explicativa, incluso heurística.

Bunge (1985) precisa que la finalidad de la modelización en ciencia es comprender la realidad. Martín (2013: 43) delimita el concepto de modelo en el ámbito de la supervisión educativa:

Un modelo es una forma de representar las relaciones y vínculos estructurales de los diferentes conceptos e ideas claves que están presentes en el entramado teórico que intentan reflejar. Son elaboraciones con un mayor nivel de concreción que replican y visualizan los elementos comprendidos tanto en los principios y supuestos de naturaleza teórica que guían y dan sentido a las actuaciones, como las soluciones concretas que se adoptan en las prácticas supervisoras y que de una u otra forma contribuyen a la validación y acrecentamiento de sus bases teóricas. La combinación, por tanto, de planteamientos teóricos y prácticos y de componentes reales y utópicos singularizan la orientación y forma de proceder de los diferentes modelos de la supervisión educativa. 
JESÚS MANUEL LUCENDO PATIÑO

\subsection{Trabajo de campo}

Como fuente de información se utiliza la técnica de entrevista individual en profundidad, intensiva y estructurada (Gaínza, 2006; Pérez Juste, Galán y Quintanal, 2011). La justificación de la elección de esta técnica de investigación concreta, entrevista en profundidad, se fundamenta en las ventajas atribuidas por Palacios y Rubio (2003) a esta herramienta escogida que concuerdan con el plan de esta investigación: pertinente cuando se busca información de profesionales, cuando se requiere información muy compleja y detallada, permite conocer los temas en profundidad, no existe influencia de opiniones grupales y tiene carácter holístico. En palabras de estos autores, la entrevista ofrece «una imagen holográmica de la sociedad en movimiento» (p. 15).

Para la realización de esta entrevista estructurada, se elabora una guía directriz que contienen la contextualización de la investigación, objetivos, cuestiones metodológicas y treinta ítems que describen las variables de estudio sobre las que se realiza la investigación. En concreto, se incorporan cinco cuestiones relativas a la perspectiva que existe de la Educación de Personas Adultas y la Inspección de Educación desde la Universidad; su presencia en las guías didácticas de los diversos planes de estudio; relación y vínculos entre la Universidad, los centros de adultos y los Servicios de Inspección de Educación; existencia de formación de postgrado, másteres o doctorados en estos ámbitos; grupos de investigación constituidos en estas áreas de estudio; publicaciones y repositorios. Igualmente, se pretende indagar sobre modelos y teorías del corpus de la Didáctica y Organización Escolar relevantes; experiencias de buenas prácticas en Educación de Personas Adultas, y mejoras e innovaciones en enseñanza de adultos y supervisión cuya implementación repercutiría significativamente sobre la calidad educativa. También se incluyen preguntas sobre los modelos de supervisión educativa que mejor responden a las demandas y necesidades de la sociedad actual; sus principales funciones, finalidades y ámbitos de actuación; técnicas y procedimientos de inspección; estructuras de organización interna, e incidencia y repercusión de los informes de inspección. Por último, se busca información sobre la participación de la Universidad en los procesos de metaevaluación de la supervisión escolar y en la formación de los inspectores.

El proceso de realización de entrevistas se desarrolla mediante el envío de la guía a los expertos por email, previamente a las entrevistas, que se realizan en los centros de trabajo del entrevistado, excepto en un caso que tiene lugar online.

El procedimiento de análisis de la información y construcción de codificaciones y categorizaciones se organiza en dos etapas: una primera descriptiva y otra segunda interpretativa. Se secuencia en tres etapas:

1. Etapa primera: segmentación e identificación de unidades de significado y agrupación en categorías descriptivas.

2. Etapa segunda: construcción de un sistema de núcleos temáticos emergentes o metacategorías. 
JESÚS MANUEL LUCENDO PATIÑO

ANÁLISIS DE ELEMENTOS PARA LA DEFINICIÓN DE UN MODELO DE SUPERVISIÓN ESCOLAR EN EDUCACIÓN DE PERSONAS ADULTAS

3. Etapa tercera: identificación de dominios cualitativos (análisis secuencial y transversal de las metacategorías).

El análisis descriptivo de los datos se realiza mediante el conteo de frecuencias, y los vínculos entre códigos y categorías a través de redes, de las cuales se obtienen la interpretación y las conclusiones halladas.

\subsection{Participantes}

Se realiza una primera discriminación de participantes utilizando el procedimiento de muestreo no probabilístico intencional (Ávila, 2006), ya que el objetivo del estudio no es estadístico sino cualitativo exploratorio a partir de competencia experta. El proceso general de muestreo es motivado o teórico, con técnicas de muestreo de bola de nieve lineal. Baltar y Gorjup (2012) consideran esta técnica apropiada para investigaciones exploratorias, cualitativas y descriptivas, sobre todo en los estudios en los que los encuestados son pocos en número o se necesita un elevado nivel de confianza para desarrollarlas. Posteriormente, entre la primera selección, se eligió a los entrevistados mediante Coeficiente Experto, Coeficiente $\mathrm{K}, \mathrm{K}=1 / 2(\mathrm{Kc}+\mathrm{Ka})$ en el que se considera $\mathrm{Kc}$ el "Coeficiente de conocimiento" $\mathrm{O}$ información que tiene el experto acerca del tema o problema planteado y Ka el "Coeficiente de argumentación" o fundamentación de los criterios de los expertos (Cabero y Barroso, 2013), seleccionando entrevistados con puntuación k ${ }^{3}$ 0.8, lo cual situó la muestra en nueve casos. El riesgo de «sesgo de comunidad" no tiene lugar en este caso dado que el objetivo de población buscado en el estudio ya estaba delimitado a priori. Adicionalmente, esta técnica presenta entre sus ventajas la simplicidad, economía y sencillez, basándose en que la población diana cumple los tres primeros de los cuatro requisitos establecidos por Marpsata y Razafindratsimab (2010): el tamaño de la población de interés es relativamente bajo, los miembros de la población de interés son difíciles de identificar, no hay marco muestral o es muy incompleto y la distribución geográfica de la población de interés no es pública.

Una vez configurada la muestra de encuestados, se analizan las características comunes a los biogramas de todos los entrevistados: respecto a formación académica, el 66\% son doctores y el 34\% restante poseen licenciatura, con alta acreditación de formación continua, participación en proyectos de investigación y publicaciones académicas en todos los casos; con relación a la experiencia profesional, todos los expertos tienen experiencia en docencia universitaria y en otro nivel de enseñanza no universitario; han desempeñado función de inspectores de educación, salvo tres casos que han sido cargos directivos de centros de adultos o enseñanza secundaria.

\subsection{Análisis de datos}

La información obtenida de las entrevistas estructuradas se codifica y categoriza mediante CAQDAS, en concreto ATLAS.ti, versión 8.1.3 (522) para Mac, generando citas, 
categorías y codificaciones para la posterior creación de unidades redes conceptuales de datos con la información aportada, que fundamenten la creación, generación de tipologías y visualización del modelo de supervisión en educación de adultos.

A partir del análisis realizado con las herramientas Wordcruncher y la Tabla de Co-ocurrencias se genera la categorización de la siguientes metavariables:

1. Paradigmas teóricos de supervisión educativa.

2. Relación supervisión educativa y educación de personas adultas.

3. Finalidades y funciones.

4. Ámbitos de supervisión en Educación de Personas Adultas.

5. Cualidades del buen inspector y una práctica supervisora eficaz.

6. Técnicas, procedimientos e instrumentos de supervisión Temporalización.

7. Organización interna de los equipos de inspección educativa.

8. Formación específica en este ámbito: grupos colaborativos.

9. Metaevaluación del proceso de supervisión.

\section{Resultados}

Con relación a la variable 1:

Los paradigmas de supervisión con mayor frecuencia de aceptación son aquellos orientados a priorizar la evaluación, guiadores de procesos para la mejora y evaluadores del cumplimiento de objetivos rechazando modelos burocráticos y jerárquicos basados en tareas control. En la Tabla 1 se relacionan las frecuencias de significado de las categorías relativas a la valoración de los modelos de supervisión.

\section{TABLA 1}

Paradigmas de supervisión educativa

\begin{tabular}{lcccc}
\hline & CASOS/CITAS & $\%$ CASOS & NB WORDS & $\%$ WORDS \\
\hline Evaluador externo & 28 & $11,9 \%$ & 364 & $11,2 \%$ \\
\hline Asesoramiento & 17 & $7,3 \%$ & 332 & $10,3 \%$ \\
\hline $\begin{array}{l}\text { Evaluador procesos } \\
\text { intermedios }\end{array}$ & 14 & $6,0 \%$ & 184 & $5,7 \%$ \\
\hline Objetivos compartidos & 19 & $8,1 \%$ & 214 & $6,6 \%$ \\
\hline Rendición resultados & 30 & $12,8 \%$ & 492 & $15,2 \%$ \\
\hline Formativo & 16 & $6,8 \%$ & 238 & $7,3 \%$ \\
\hline Innovación y mejora & 21 & $9,0 \%$ & 162 & $5,1 \%$ \\
\hline Control normativo & 48 & $20,6 \%$ & 674 & $20,8 \%$ \\
\hline Burocrático y gestión & 41 & $17,5 \%$ & 572 & $17,7 \%$ \\
\hline$N{ }^{\circ}$ Total & 234 & $100 \%$ & 3232 & $100 \%$ \\
\hline
\end{tabular}


Algunos expertos profundizan más en esta línea, siendo partidarios de separar las funciones de inspección administrativa, laboral y similares que deben ser ejercidas por técnicos en Derecho o Economía, especializando a la Inspección de Educación en tareas de control, supervisión y liderazgo exclusivamente en el terreno de lo pedagógico. Se aboga, igualmente, por la complementariedad de modelos que conjuguen la autoevaluación interna del propio centro con evaluaciones externas mediante técnicas compartidas y complementarias. Otro denominador común encontrado entre los entrevistados es la necesidad de consenso. Todos los expertos resaltan la necesidad de buscar un amplio acuerdo social de toda la comunidad educativa, para determinar un modelo Inspección de Educación que la ubique en una posición aceptada por todos y reconocida de manera acorde a su formación y alto desempeño profesional, con una valoración y presencia social (medios de comunicación, revistas especializadas, etc.) adecuada.

\section{Con relación a la variable 2 :}

Existe poca relevancia de la Educación de Personas Adultas en la actuación del Servicio de Inspección Educativa. Entre las causas, el menor número de centros, la ausencia de conflictividad y la no obligatoriedad. Los informantes con experiencia inspectora afirman que no suele estar esta modalidad de enseñanza en los planes de actuación específicamente y es más desconocida que otras enseñanzas por los propios inspectores, pues no son comunes perfiles con experiencia previa docente en este régimen de enseñanzas.

\section{Con relación a la variable 3:}

La evaluación del centro escolar y de la práctica docente junto a la evaluación pedagógica y profesional del profesorado son las finalidades y funciones de mayor relevancia para los expertos entrevistados. En un segundo lugar, la adecuación a la normativa y la orientación a la comunidad educativa.

\section{Con relación a la variable 4:}

Los ámbitos de actuación de la Inspección de Educación que tienen más incidencia sobre la mejora en Educación de Personas Adultas son la gestión del centro por parte del equipo directivo, el funcionamiento de los órganos colegiados, análisis de documentos programáticos del centro, aspectos metodológicos y de evaluación del alumnado; en detrimento de aspectos como control de horarios, ajustes de matrícula o ausencias de personal. De la información aportada por los entrevistados se genera una red de ámbitos de actuación (Figura 1). 


\section{FIGURA 1}

Red de ámbitos de actuación de la Inspección de Educación en Educación de Personas Adultas

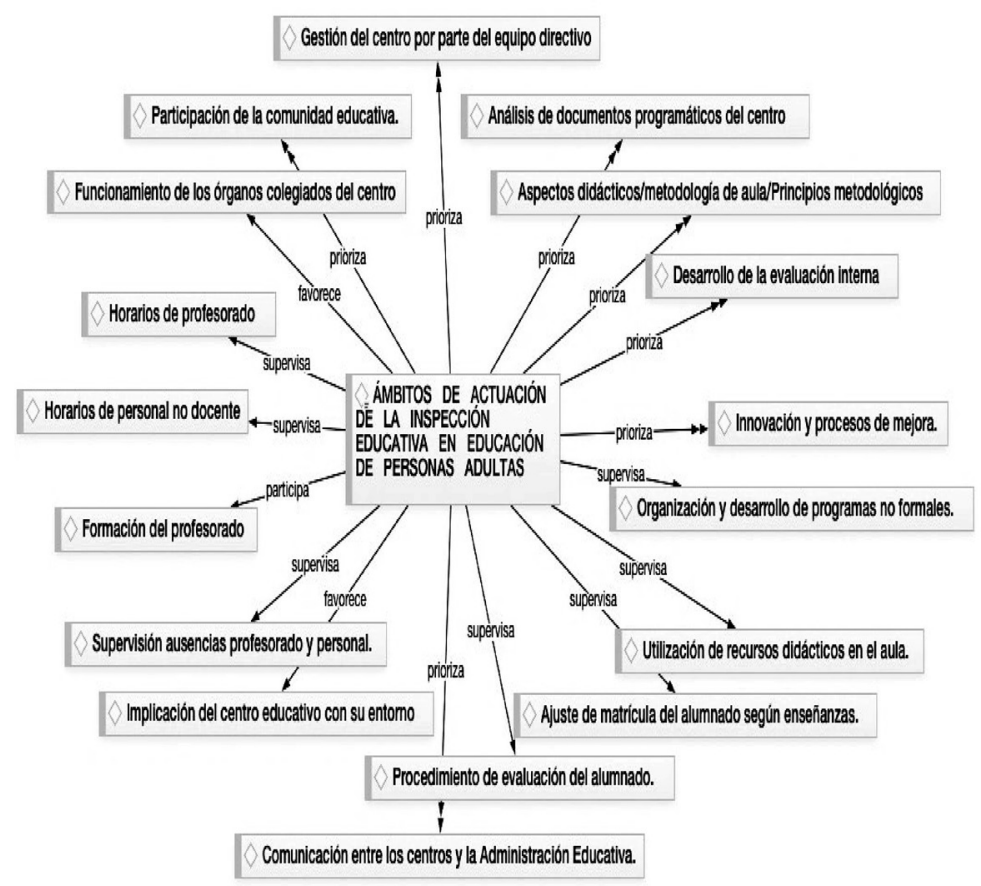

FUENTE: Elaboración propia con CAQDAS, ATLAS.ti.

El informante cuatro insiste en que "[4:34] las actuaciones de inspección deben regirse siempre por una planificación coordinada y estructurada", cuestión corroborada por el resto de los expertos. Existe coincidencia en que las enseñanzas no formales deben ser objeto de supervisión, inspeccionando especialmente en ellas su funcionalidad y conexión con el entorno del centro, demanda real de alumnado y eficacia, así como los contenidos desarrollados y la metodología docente adaptada al perfil del alumnado.

\section{Con relación a la variable 5:}

Las cualidades más valoradas en un buen inspector son la capacidad de escucha, el trabajo en equipo, habilidades comunicativas, conocimiento legislativo y aspectos éticos. En un segundo término valoran los entrevistados la capacidad de innovación, el control de conflictos, la generación de confianza y el control emocional. Es 
denominador común no otorgar importancia a la variable "domino de una materia docente» para el ejercicio inspector. La informante uno defiende que "[1:19] el trabajo de inspector debería ser en equipo siempre, refrendado por un equipo".

\section{Con relación a la variable 6:}

La técnica de supervisión más valorada por todos los participantes es la visita a centros, habituales e incidentales. La entrevista en el centro docente (no en sede de Inspección, que tiene connotaciones negativas para la mayoría de los expertos) y la participación en reuniones de trabajo y órganos colegiados ocupan un segundo lugar. Otras técnicas como cuestionarios, diario de notas, videoconferencia o revisión de materiales de profesor y alumno son poco valoradas, según se presenta en la red (Figura 2) elaborada.

\section{FIGURA 2}

Procedimientos y técnicas de la Inspección de Educación en Educación de Personas Adultas

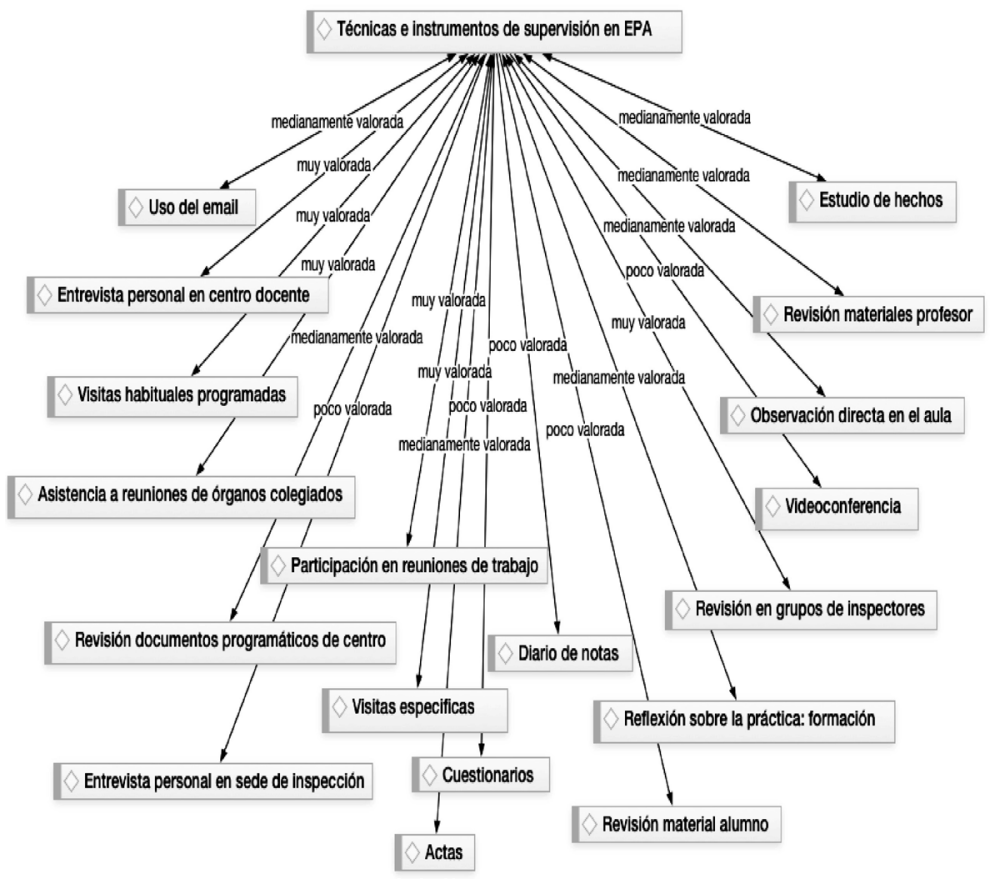

FUENTE: Elaboración propia con CAQDAS, ATLAS.ti. 
Consideran como óptima la visita de inspección a centros de adultos con una periodicidad entre trimestral y mensual y la periodicidad de Planes de Inspección Generales idónea bianual o superior mientras que para los provinciales es anual. Se considera necesario disponer de guías detalladas elaboradas por la Administración Educativa para la supervisión de enseñanzas de adultos que deben ser conocidas por los inspectores, el equipo directivo y el profesorado. Existe coincidencia en los entrevistados en resaltar la importancia de los informes de Inspección, que deben estar bien organizados, digitalizados y archivados con indexación y preferentemente protocolizados. Se otorga una alta valoración en la transcendencia de los informes de inspección para el centro educativo y el profesorado decreciendo la relevancia para el alumnado y la sociedad en general. Es común la opinión de que los informes de inspección deberían tener más difusión y ser más conocidos.

Los medios más adecuados para la comunicación con la administración y los centros educativos son la emisión de informes, el email, la entrevista presencial, la organización de seminarios y reuniones, la asistencia a órganos colegiados y la disposición de una intranet colaborativa. Sería necesario un mayor acercamiento de los inspectores a todos los sectores de la comunidad educativa, generalizando la relación y comunicación preferente con equipos directivos.

\section{Con relación a la variable 7 :}

Se encuentra preferencia por un modelo de organización interna de la Inspección de Educación basado en demarcaciones geográficas con inspector de referencia de centro, rechazando estructuras organizativas basadas en materias de conocimiento o procedencia de los inspectores. La tabla 2 muestra la transcripción de frecuencias de significado de las categorías encontradas respecto a la organización interna de la Inspección de Educación.

\section{TABLA 2}

Modelos de organización interna de la IE

\begin{tabular}{lcccc}
\hline & CASOS / CITAS & \% CASOS & NB WORDS & \% WORDS \\
\hline Demarcación geográfica & 13 & $13,4 \%$ & 282 & $11,2 \%$ \\
\hline Inspector de referencia & 21 & $21,9 \%$ & 197 & $10,3 \%$ \\
\hline Materias & 14 & $14,6 \%$ & 192 & $5,7 \%$ \\
\hline Equipo de inspección & 18 & $18,8 \%$ & 237 & $6,6 \%$ \\
\hline Inspector de adultos & 30 & $31,3 \%$ & 296 & $15,2 \%$ \\
\hline$N{ }^{\circ}$ Total & 96 & $100 \%$ & 1616 & $100 \%$ \\
\hline
\end{tabular}

Otra idea común expuesta por los entrevistados incide en favorecer modelos de organización que incrementen la democratización, participación en la toma de 
decisiones y que potencien el consenso entre todos los sectores implicados en la Educación de Personas Adultas.

\section{Con relación a la variable 8:}

Los expertos consideran la formación inicial y continua de los inspectores como un parámetro esencial para el buen ejercicio profesional. Son partidarios de procedimientos de formación interna mediante grupos colaborativos o procedimientos de investigación-acción. También demandan la necesidad de ofertar formación específica universitaria sobre supervisión educativa, especialmente como especialización de postgrado.

\section{Con relación a la variable 9:}

Los entrevistados muestran preferencia por un modelo de metaevaluación en Inspección de Educación cuya finalidad principal sea la eficacia de la tarea y la mejora de la actuación supervisora; existe discrepancia entre los expertos respecto a que pueda tener incidencia sobre una posible carrera profesional dentro del Servicio de Inspección y la asignación de responsabilidades. El procedimiento más valorado es la coevaluación por comisión de inspectores entre quienes tienen experiencia en inspección; en menor medida, metaevaluación externa estandarizada (más valorada por los otros tres entrevistados sin ejercicio profesional supervisor) e informes de Inspector Jefe Provincial y Inspección Central y General. La Tabla 3 muestra las frecuencias de las categorías totales relativas a esta variable.

\section{TABLA 3}

Metaevaluación de la actuación inspectora

\begin{tabular}{lcccc}
\hline & CASOS / CITAS & $\%$ CASOS & NB WORDS & $\%$ WORDS \\
\hline Inspección Central & 10 & $15,9 \%$ & 82 & $18,3 \%$ \\
Inspector provincial & 16 & $25,4 \%$ & 103 & $22,9 \%$ \\
Comunidad educativa & 9 & $14,3 \%$ & 62 & $13,8 \%$ \\
Equipo de inspección & 14 & $22,2 \%$ & 107 & $23,8 \%$ \\
Evaluación externa & 14 & $22,2 \%$ & 95 & $21,2 \%$ \\
\hline$N^{\circ}{ }^{\circ}$ Total & 63 & $100 \%$ & 449 & $100 \%$
\end{tabular}

Por último, acorde con los objetivos propuestos, se ha generado una red conceptual (Figura 3) que integra todos los elementos, a partir de los cuales se pueda estructurar y fundamentar la generación del modelo de supervisión en Educación de Personas Adultas en función de las aportaciones informadas por los expertos para la elaboración del modelo buscado. 


\section{FIGURA 3}

Elementos y nodos de relación para la elaboración de un modelo de Supervisión en Educación de Personas Adultas

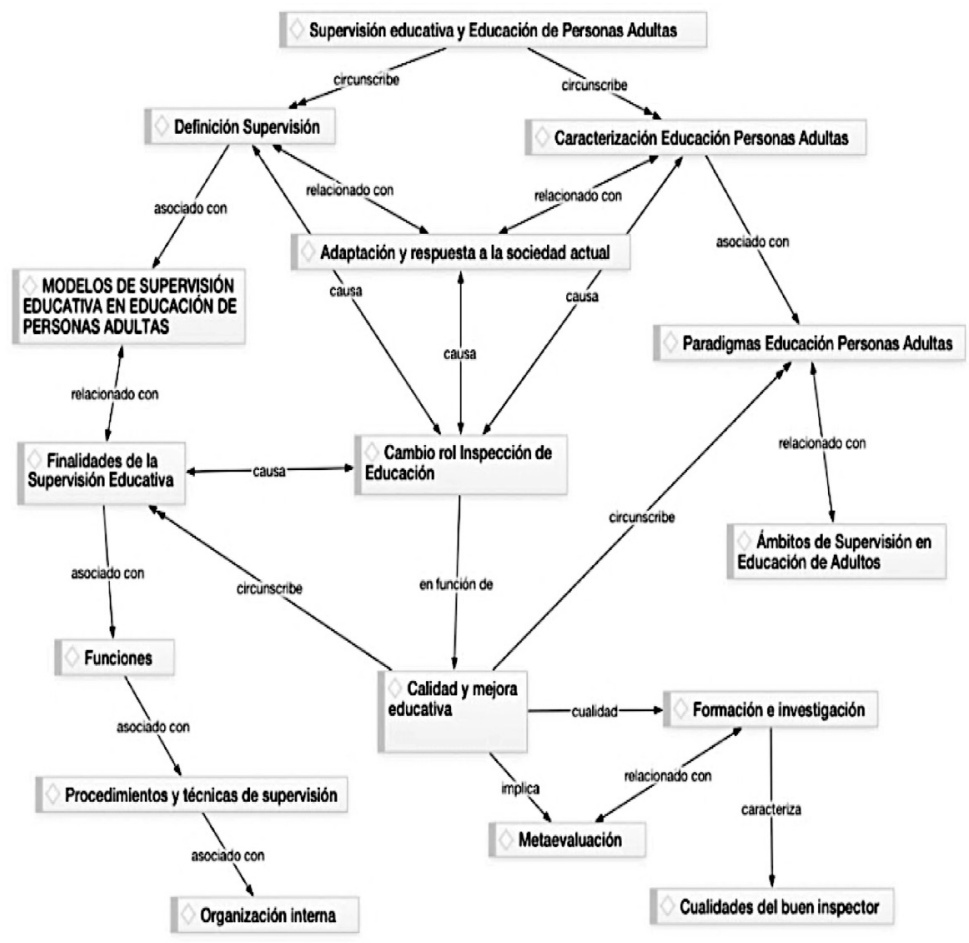

FUENTE: Elaboración propia con CAQDAS, ATLAS.ti

Supone este paradigma un cambio cualitativo en la conceptualización de la supervisión en Educación de Personas Adultas puesto que se da relevancia e importancia a un ámbito de supervisión, la Educación Permanente, hasta ahora considerado menor frente a otras enseñanzas (Primaria, Secundaria, etc.).

En segundo lugar, supone un cambio de enfoque en la supervisión de Educación de Personas Adultas situando como piedra angular el trabajo por objetivos compartidos por toda la comunidad educativa y liderado, supervisado, asesorado y evaluado por los organismos de supervisión educativa en equipo. Se introducen nuevas funciones para la Inspección de Educación, Planificación, Coordinación y Liderazgo Educativo, posicionándola circularmente al principio del hecho educativo y no solo al final, como tradicionalmente se ha considerado con las funciones clásicas de supervisión, control, asesoramiento y evaluación. 
Se rompe la perspectiva de focalización en tareas de la mayor parte de los modelos establecida hasta ahora; se abandona la idea del inspector de referencia común actualmente por la del equipo de referencia con la imposición de dinámicas de trabajo en equipo, participativas y colaborativas con carácter ineludible; se impone la necesidad de proximidad a la comunidad educativa de los supervisores educativos, con lo que se mejorará la percepción de la Inspección de Educación por parte de la comunidad escolar y profesionales docentes; se pone en valor su actuación y adquiere mayor relevancia de cara a la sociedad, posibilitando mayor repercusión funcional directa en la enseñanza de adultos al trabajo de inspector. El supervisor en este modelo se ve obligado a salir del despacho en continua interacción con el entorno social y las comunidades educativas de su zona de educación de adultos.

\section{DISCUSIÓN Y CONCLUSIONES}

De los resultados obtenidos se concluye la necesidad de renovar y adaptar a las nuevas necesidades de aprendizaje y perfiles educativos de los adultos los modelos de supervisión en la línea expuesta por autores como Aguerrondo (2013: 26) «Las reformas educativas orientadas hacia la equidad requieren de cambios desde el rol tradicional de carácter administrativo de las personas supervisoras al de gestores activos de la mejora escolar» o Miranda (2002: 1) que afirma que «actualmente, la supervisión escolar no puede concebirse sin relacionarla con el desarrollo y la mejora de los centros".

Se ha constatado científicamente la demanda que los entrevistados y expertos solicitan a la actual Inspección de Educación como cuerpo técnico y especializado dotado de gran prestigio para superar las antinomias y reminiscencias derivadas de su historia, proponiéndoles el reto de actualizar sus funciones basadas en el control, la supervisión y la evaluación (ocupaciones a las que no debe renunciar), con la incorporación de nuevas finalidades de liderazgo pedagógico, la innovación y la mejora, el asesoramiento para la calidad educativa, la participación en la planificación escolar, etc.; se le demandan nuevas cualidades, habilidades y competencias de actuación (dominio TIC, destrezas comunicativas, habilidades de planificación, etc.) al supervisor de educación; se introduce la necesidad de más recursos humanos en esta función; mayor responsabilidad y riesgo en la toma de decisiones del inspector que pasa a tener atribuciones ejecutivas y no solo de informar y proponer como en los modelos en vigor; el desafío de una mayor cercanía y cooperación con los centros, de situarse al lado de las comunidades educativas y no por delante; de guiar, en definitiva, desde una perspectiva experta y técnica pedagógica a los sistemas educativos y los centros hacia las respuestas y actuaciones de mayor calidad frente a los novedosos y cambiantes aprendizajes que la permanente evolución de nuestra sociedad actual impone cada día.

El nuevo modelo propuesto otorga a la Inspección de Educación una actitud y competencia proactiva frente a las demandas educativas de la sociedad actual y no solo reactiva frente a las peticiones de la administración o los centros de 
educación de personas adultas, en consonancia con los presupuestos de partida, los planteamientos y objetivos diseñados en esta investigación que buscan adaptar la supervisión educativa en Enseñanzas de Adultos a las necesidades de aprendizaje y realidades cambiantes y versátiles de la sociedad del siglo XXI.

\section{REFERENCIAS BIBLIOGRÁFICAS}

Aguerrondo, I. (2013). El rol de la supervisión educativa en la gestión de las políticas públicas. Educar, 49 (1), 13-27.

Alcalá, M. L. (2016) ¿Debe ser la inspección impulsora de la innovación en los centros educativos? Avances en Supervisión Educativa, 26. doi: https://doi.org/10.23824/ase.v0i26.578.

Alonso, M. J. (2002). La investigación en educación de personas adultas en el estado español: logros y desafíos. En F. Sanz Fernández (Coord.). La Educación de Personas Adultas entre dos siglos: historia pasada y desafíos de futuro (pp. 323-350). Madrid: UNED.

Ávila, H. L. (2006). Introducción a la metodología de la investigación. México: Eumed Ediciones.

Baltar, F. y Gorjup, M. (2012). Muestreo mixto online: Una aplicación en poblaciones ocultas. Intangible Capital, 8 (1), 123-149.

Bolívar, A. (2000). Los centros educativos como organizaciones que aprenden. Promesa y realidades. Madrid: La Muralla.

Bradley, L. H. (1993). Total Quality Management for Schools. Lancaster-Pennsylvania: Technomic Publishing Company INC.

Bunge, M. (1985). Teoría y realidad. Barcelona: Ariel.

Cabero, J. y Barroso, J. (2013). La utilización del juicio de experto para la evaluación de TIC: el coeficiente de competencia experta. Bordón, 65 (2), 25-38. doi: http://dx.doi. org/10.13042/brp.2013.65202.

Camacho, A. (2014). Funciones y quehaceres de los inspectores de educación en Baleares. Un estudio de casos. (Tesis doctoral). Recuperado de http://tdx.cat/bitstream/ handle/10803/134797/ACP_TESI.pdf?sequence=1.

Campos, B. (2017). Inspección educativa y calidad institucional. Madrid: Universitas.

Cardona, J. (2013). Epistemología del saber docente. Madrid: Universidad Nacional de Educación a Distancia.

Casanova, M.A. (2015). La supervisión, eje del cambio en los sistemas educativos. REICE. Revista Iberoamericana sobre Calidad, Eficacia y Cambio en Educación, 13 (4), 7- 20. Recuperado de: http://www.rinace.net/reice/numeros/arts/vol13num4/art1.pdf.

Consejo de la Unión Europea (2011). Resolución del Consejo sobre un plan europeo renovado de aprendizaje de adultos. Diario Oficial de la Unión Europea 2011/C 372/01. Recuperado de https://eur-lex.europa.eu/legal-content/ES/TXT/PDF/?uri=CELEX:32011G1220 (01)\&from $=\mathrm{ES}$.

Domingo, J. (2001). Asesoramiento al centro educativo. Colaboración y cambio en la institución. Barcelona: Octaedro. 
JESÚS MANUEL LUCENDO PATIÑO

ANÁLISIS DE ELEMENTOS PARA LA DEFINICIÓN DE UN MODELO DE SUPERVISIÓN ESCOLAR EN EDUCACIÓN DE PERSONAS ADULTAS

Ehren, M. C. M. (2017). Mecanismos y modelos de inspecciones eficaces en el contexto europeo. En Vázquez-Cano, E. (Coord.). La inspección y supervisión de los centros educativos (pp. 451-476). Madrid: UNED.

Estefanía, J.L. (2014). Retos de la Inspección ante los cambios en educación. Avances en Supervisión Educativa (22), ADIDE. Recuperado de: https://avances.adide.org/index.php/ ase/article/view/68.

European Commission (2015). Assuring Quality in Education: Policies and Approaches to School Evaluation in Europe. Eurydice Report. Luxembourg: Publications Office of the European Union. doi: https://doi.org/10.2797/678.

European Commission (2017). An in-depth analysis of adult learning policies and their effectiveness in Europe. Luxembourg: Publications Office of the European Union. doi: https://doi.org/10.2767/076649.

Fernández Enguita, M. (2016). La educación en la encrucijada. Madrid: Fundación Santillana.

Fernández Pérez, M. (1978). Modelos conceptuales de las ciencias humanas y su aplicación a las ciencias de la educación. En J. Escolano, Epistemología y Educación (pp. 122-153). Salamanca: Sígueme.

Fullan, M. (2002). Los nuevos significados del cambio. Barcelona: Octaedro.

Gaertner, H. y Pant, H. A. (2011). How valid are school inspections? Problems and strategies for validating processes and results. Studies in Educational Evaluation, 37 (23), 85-93.

Gaínza, A. (2006). La entrevista en profundidad individual. En M. Canales, Metodologías de investigación social (pp. 219-261). Santiago: Lom Ediciones.

García, B. y Zendejas, L. (2008). Hacia un nuevo modelo de supervisión escolar para las primarias mexicanas. México: Instituto Nacional para la Evaluación de la Educación.

García, J. y Martín, A. (2007). Visiones, revisiones y reversiones de la educación de adultos: aportaciones de la educación de adultos al sistema social de educación. EFORA-Revista de Educación, (1), 35-62. Recuperado de http://campus.usal.es/ efora/revista_1/articulos_rev_1/articulos_rev_1_pdf/n1_01_carrasco_garcia.pdf.

Glaser, B. G. y Strauss, A. L. (1967). The Discovery of Grounded Theory. Strategies for Qualitative Research. New York: Aldine.

Gento, S. (2001). La Inspección de Educación en el Marco Español y Europeo como impulsora de la Calidad Educativa. En Congreso nacional sobre liderazgo en el sistema educativo español (pp. 95-129). Córdoba: Actas del Congreso.

Gento, S. (2004). Supervisión educativa. Madrid: Sanz y Torres.

IGEN, Inspection Générale de l'Éducation Nationale (2016). Rapport d'activité. Recuperado de http://cache.media.education.gouv.fr/file/2016/65/6/Rapport_IGEN2016-V2-19-09-2017_815656.pdf.

Jiménez, R. (2009). Educación de personas adultas en el marco del aprendizaje a lo largo de la vida. Madrid: UNED.

Lancho, J. (2007): El círculo vicioso de la Educación de Adultos española. Revista de Educación y Formación Continua de Personas Adultas, 1 (1).Universidad de Salamanca. Recuperado de http://campus.usal.es/ efora/revista_1/articulos_rev_1/articulos_rev_1_pdf/ n1_01_lancho.pdf. 
JESÚS MANUEL LUCENDO PATIÑO

ANÁLISIS DE ELEMENTOS PARA LA DEFINICIÓN DE UN MODELO DE SUPERVISIÓN ESCOLAR EN EDUCACIÓN DE PERSONAS ADULTAS

López-Barajas, E. y Sarrate, M. L. (2002). La Educación de Personas Adultas: reto de nuestro tiempo. Madrid: Dykinson.

MacBeath, J.; Schratz, M.; Meuret, D. y Jacobsen, L. B. (2000). Self-evaluation in European Schools. A story of change. London. Routledge-Falmer.

Marpsata, M. y Razafindratsimab, N. (2010). Survey methods for hard-to-reach populations: introduction to the special issue. Methodological Innovations Online, 5 (2): 3-16.

Martín, E. (2013). Dirección y supervisión de centros formativos. Bloque temático II: Supervisión. Madrid: UNED.

Matthews, P. y Sammons, P. (2004). Improvement through Inspection. London: OFSTED.

Mayorga, A. (2000). La Inspección Educativa. Siglo y medio de la Inspección Educativa en España 1849-1999. Madrid: Ediciones Santillana.

Medina, O. (2012). Apuntes para una historia de la Educación de Adultos. Las Palmas de Gran Canaria. Universidad de Las Palmas de Gran Canaria. Recuperado de: https://acceda. ulpgc.es/bitstream/10553/19967/5/Apuntes_historia_Educacion_Adultos.pdf.

Miguel, V.; Aguilar, E. V.; Gómez, A. P.; Bejarano, R. A.; De la Riva, R. D.; Pose, F. J. V.; y González, R. P. (2018). La supervisión educativa en Europa: Sistemas educativos y modelos de inspección educativa. Journal of Supranational Policies of Education (JoSPoE), (7), 131-161. doi: http://dx.doi.org/10.15366/jospoe2018.7.

Mills-Scofield, D. (2012). It's not just semantics: managing outcomes vs. outputs. Harvard Business Review. Recuperado de https://hbr.org/2012/11/its-not-just-semantics-managingoutcomes.

Miranda, E. (2002). La supervisión escolar y el cambio educativo. Un modelo de supervisión para la transformación, desarrollo y mejora de los centros. Profesorado, Revista de Currículum y Formación del Profesorado, 6 (1-2), 1-15.

OFSTED. Office for Standards in Education, Children's Services and Skill (2017a). Ofsted strategy 2017-2022. Recuperado de https://assets.publishing.service.gov.uk/government/ uploads/system/uploads/attachment_data/file/648212/Ofsted_strategy_2017-22.pdf. https://www.gov.uk/government/uploads/system/uploads/attachment_data/file/648212/ Ofsted_strategy_2017-22.pdf.

OFSTED. (2017b). School inspection handbook. Recuperado de https://www.gov.uk/government/publications/school-inspection-handbook-from-september-2015.

Palacios, S. P. y Rubio, K. L. (2003). La entrevista en profundidad: teoría y práctica. México. Universidad Autónoma de Tamaulipas.

Pérez Juste, R.; Galán González, A. y Quintanal Díaz, J. (2011) Métodos y Diseños de Investigación en Educación. Madrid: UNED.

Ramírez, E. (2017). Historia de la Inspección de Educación en España: 1812-1978. En E. Vázquez-Cano (Coord.). La Inspección y Supervisión de los centros educativos (pp. 36-65). Madrid: UNED.

Rul, J. (2013). Problemas y retos de la inspección de educación. Educar, 49 (1): 29-48. Barcelona: Universitat Autònoma de Barcelona.

Rumbo, B. (2016). Problemas y retos de la educación de las personas adultas. Educar, 52 (1), 93-106.doi: http://dx.doi.org/10.5565/rev/educar.707. 
JESÚS MANUEL LUCENDO PATIÑO

ANÁLISIS DE ELEMENTOS PARA LA DEFINICIÓN DE UN MODELO DE SUPERVISIÓN ESCOLAR EN EDUCACIÓN DE PERSONAS ADULTAS

Salgado, F. J. (2009). Funcións da inspección: aspectos curriculares e de desenvolvemento profesional na comunidade autónoma de Galicia. Tesis doctoral. Universidad de Santiago de Compostela. Santiago de Compostela.

Sandoval, C. (1997). Investigación cualitativa. Programa de especialización en teoría, métodos y técnicas de investigación social. Medellín: Universidad de Antioquía.

Sanz Fernández, F. (1998). Perspectivas de la educación de adultos en una sociedad globalizada. Revista Española de Educación Comparada, 4, 69-100. Recuperado de http:// revistas.uned.es/index.php/REEC/article/view/7250.

Secadura, T. (2014). Fortalezas y debilidades de la organización y funcionamiento de la Inspección de Educación. Avances en Supervisión Educativa, (21). Recuperado de https:// avances.adide.org/index.php/ase/article/view/77.

Secadura, T. (2017). La Inspección Educativa ante el siglo XXI. Retos y propuestas de mejora. En E. Vázquez-Cano (Coord.). La Inspección y Supervisión de los centros educativos (pp. 501-520). Madrid: UNED.

Sevillano, M. L. y Vázquez-Cano, E. (2015). Modelos de investigación en contextos ubicuos y móviles en educación superior. Madrid: McGrawHill.

SICI. Standing International Conference of Inspectorates (2016). Strategic plan 2016/2020. Recuperado de http://www.sici-inspectorates.eu/getattachment/b38cc7c8-0680-406289f3-d5f5b29d1a31.

Silva, B. P. (2013). El papel de la inspección escolar en la mejora de los resultados educativos. Educar, 49, 67-82. Recuperado de www.redalyc.org/service/redalyc/downloadPdf/3421/342130840005/6.

UNESCO. United Nations Educational, Scientific and Cultural Organization (2015a). Incheon Declaration Education 2030: Towards inclusive and equitable quality education and lifelong learning for all. Incheon, Corea. World Declaration Forum. Recuperado de http:// unesdoc.unesco.org/images/0023/002338/233813M.pdf.

UNESCO. (2015b). Rethinking Education. Towards a global common good? Recuperado de http://unesdoc.unesco.org/images/0023/002325/232555e.pdf.

unEsCO. (2016). Third Global Report on Adult Learning and Education. Hamburg: Institute for Lifelong Learning UNESCO.

Vázquez- Cano, E.; Arévalo, J. J. y Gómez de Agüero, J. L. (2011). El desarrollo de cartas de servicios en la inspección de educación. Factor de calidad en la atención al ciudadano. Avances en Supervisión Educativa, (15). Recuperado de https://avances.adide.org/index. php/ase/article/view/487/328.

Vázquez-Cano, E. (2017). La innovación educativa: asesoramiento y supervisión. En E. Vázquez-Cano (coord.). La inspección y supervisión de los centros educativos (pp. 419-449). Madrid: UNED. 